\title{
ABUNDANCE VARIATIONS OF TRACERS AND THEIR EFFECTS ON OUR DETERMINATION OF MOLECULAR CLOUD STRUCTURE
}

\author{
Paul F. Goldsmith \\ Five College Radio Astronomy Observatory \\ Department of Physics and Astronomy \\ University of Massachusetts, Amherst MA 01003
}

Our understanding of the molecular phase of the interstellar medium is critically dependent on use of various lines from different molecular species to trace this dense material. As our knowledge of molecular clouds becomes more refined, and we pursue in detail issues of molecular cloud structure, stability, and how star formation depends on and affects the molecular gas, it is appropriate to examine the basis by which we determine the morphology of clouds, their density, and other key parameters. This is obviously a major undertaking, well beyond the scope of the short presentation at this conference, so I will concentrate on one very basic, but critical issue, which is that of abundance variations of tracers of density and molecular column density which are widely used to delineate the denser portions of all types of molecular clouds. In this summary, I will first highlight some of the apparent indications of significant variations of abundance within individual clouds, as a way of indicating some potential dangers and the importance of the molecular tracer selected. I will also briefly suggest how such variations may be themselves important diagnostics of cloud structure and evolution.

The observation of abundance variations of different molecular species is certainly not new; while there is reasonable consistency for the fractional abundance of different species in various clouds, there are also clear differences. This issue is discussed in greater detail by Irvine, Goldsmith, and Hjalmarson (1987), Federman, Huntress, and Prasad 1990, and Irvine (1990). Comparison of the abundance of various molecular species requires properly analyzing molecular excitation and radiative transfer, a task which is made even more difficult when studying different clouds where the antenna beam is subtending different linear dimensions and thus likely averaging over regions with greatly varying conditions. Determining abundances within an individual cloud should be somewhat less susceptible to this type of error than is comparison of different clouds. Even when restricting observations to a single cloud, however, the variations of conditions from one line of sight to another make unambiguous determination of even such a basic quantity as column density of a particular species quite difficult.

We might expect the abundance of a particular molecular species within a given cloud or cloud complex to exhibit significant variations as a result of radiation field, density, temperature, and other even less well-understood conditions such as the passage of shock waves. This type of dependence is borne out in a number of cases where the structure of the cloud permits isolating variations in (hopefully) a single parameter. For example, many studies have been made of the correlation of 
${ }^{13} \mathrm{CO}$ column density with total column density traced by visual extinction (e.g. Dickman 1978). For moderate extinctions it is clear that the fractional abundances of ${ }^{13} \mathrm{CO}$ and $\mathrm{C}^{18} \mathrm{O}$ depend on the extinction characterizing the line of sight (cf. Goldsmith et al. 1980; Frerking, Langer, and Wilson 1982). Nevertheless, these same isotopes are widely used as tracers of total line of sight column density, with a single constant of proportionality employed for an entire cloud, or region being studied (e.g. Arquilla and Goldsmith 1983; Langer et al. 1987). While this is understandable in terms of ease of analyzing a map, and since it is difficult to accurately correct for abundance variations predicted in terms of a model, we must keep in mind that the assumption of a single fractional abundance for a specific molecular species even in a single cloud may be seriously misleading. From the perspective of theoretical models of interstellar cloud chemistry, these variations should be larger for less abundant species, such as the "high dipole-moment" molecules used to trace dense regions in clouds (Graedel, Langer, and Frerking 1982). Thus, cloud structure may appear to be very different depending on which tracer is used, even when effects of excitation and radiative transfer are correctly accounted for.

Major differences in cloud structure indicative of variations in fractional abundance are seen in the quiescent dark cloud L134N (L183). This region has been mapped by Swade (1989) in six molecules. No unambiguous explanation is available, but significant variations, particularly between the distributions of CS, $\mathrm{H}^{13} \mathrm{CO}^{+}, \mathrm{SO}$, and $\mathrm{NH}_{3}$ are apparent. A similar effect has been recognized in the TMC1 cloud, which is discussed in detail by Olano, Walmsley, and Wilson (1988). Again, in a region apparently devoid of star formation, significant abundance gradients in the case of $\mathrm{NH}_{3}$ and cyanopolyynes are found.

In the case of clouds with massive star formation, very large variations in the abundance of many species have been found. Although it is not easy to directly determine the total $\mathrm{H}_{2}$ column density, relative abundance variations can be determined with some confidence. For example, in the region surrounding the HII region complex Sgr B2(N) having a luminosity of $\approx 10^{7} \mathrm{~L}_{0}$, Vogel Genzel and Palmer (1987) find a fractional abundance of $\mathrm{NH}_{3} \cong 10^{-5}$, an enhancement of a factor of 100 to 1000 relative to the general cloud material. These authors, and others, have suggested that this may be the result of evaporation of molecules frozen on grain surfaces in regions warmed by newly-formed stars. On the other hand, Zhou Evans, and Mundy (1990) have found evidence for an order of magnitude reduction in the fractional abundance of $\mathrm{NH}_{3}$ in the region immediately surrounding the young stars in NGC 2071. Given these highly disparate results, it is difficult to have unbridled confidence in the ability of ammonia to trace regions of high mass star formation in molecular clouds.

In Figure $1 \mathrm{I}$ show maps of the Sgr B2 region in SO and HNCO (taken from Lis and Goldsmith, 1990) which highlight the difference found on relatively large scales in the relative abundance of various molecular species. These species have been found by Goldsmith et al. (1987) to exhibit significant abundance differences on a much smaller scale, between the Middle and Northern cores in Sgr B2.

While it is difficult to eliminate completely the issue of nonuniform excitation, it seems likely that the evolutionary state of a dense cloud core with an embedded massive protostar has a major effect on the molecular content of the surrounding material, as suggested by Vogel, Genzel and Palmer and by Goldsmith et al. 


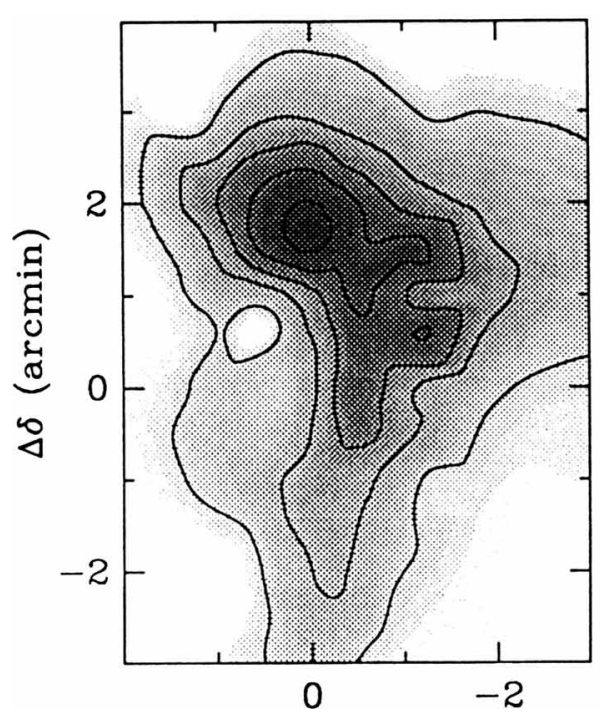

$\Delta \alpha(\operatorname{arcmin})$

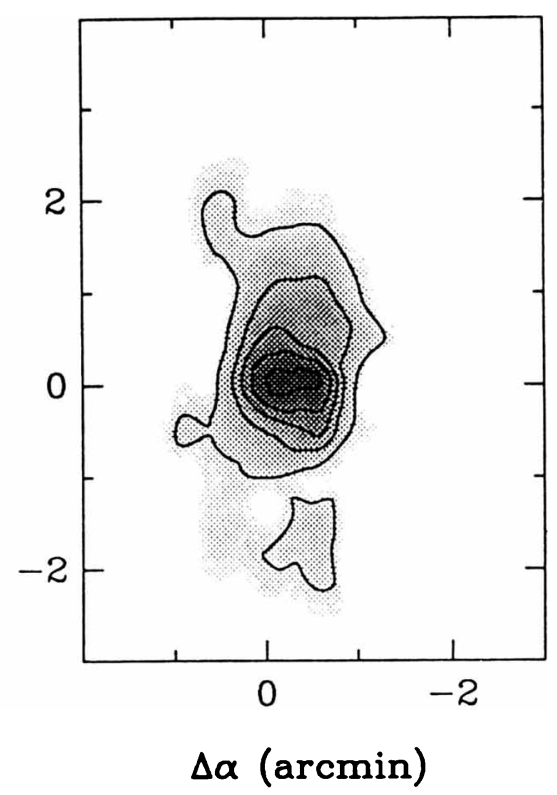

FIG. 1 - Maps of integrated intensity of HNCO $5_{05}-4_{04}$ (left) and SO $2_{3}-1_{2}$ (right) in Sgr B2 made with FCRAO antenna. The coordinates of the central position are $\alpha=17^{\mathrm{h}} 44^{\mathrm{m}} 10^{\mathrm{s}} .5, \delta=-28^{\circ} 22^{\prime} 05^{\prime \prime}$.

Within the central portion of the Orion molecular cloud are found components having different spatial and kinematic structure (extended ridge, compact ridge, hot core, and plateau; cf. Johansson et al. 1984; Blake et al. 1987), which also have significantly different chemical composition (Plambeck and Wright 1988).

Detailed, multi-transition studies of $\mathrm{HC}_{3} \mathrm{~N}$ have yielded the $\mathrm{H}_{2}$ density distribution in Sgr B2, which does not closely resemble either of the above maps in appearance, but rather follows the general distribution of $\mathrm{C} 18 \mathrm{O}$ found by $\mathrm{Lis}$ and Goldsmith (1989). Thus, there appear to be highly significant parsec-scale abundance variations which can seriously affect our perception of the overall cloud structure. The origin of these variations is quite uncertain at the present time. The enhanced abundance of $\mathrm{HOCO}^{+}$relative to predictions of standard ion-molecule astrochemical models led Pineau des Forets, Flower and Roueff (1989) to postulate its production via destruction of $\mathrm{HCO}^{+}$in $\mathrm{MHD}$ shocks. The primary $\mathrm{HNCO}$ peak seen in Figure 1, and also a secondary maximum in $\mathrm{HC}_{3} \mathrm{~N}$ $\mathrm{J}=12-11$, coincide with the strongly peaked $\mathrm{HOCO}^{+}$emission mapped by Minh, Irvine, and Ziurys (1988). While it is desirable to carry out more complete modeling of the effects of shocks on cloud chemistry, the lack of any apparent driving source (HII region or massive young star) as well as of any effect on the line profiles makes this explanation problematic for the "2' North" peak in Sgr B2.

Another cloud whose structure seems to be highly dependent on the molecular tracer used is NGC $2071 \mathrm{~N}$. This region was studied by Iwata, Fukui, and Ogawa (1988) who found very different appearance as traced by $\mathrm{NH}_{3}$ and $\mathrm{C}^{18} \mathrm{O}$. In a recently-completed project, Goldsmith et al. (1990) have obtained maps with the 
FCRAO antenna of three carbon monoxide isotopes, as well as of CS $\mathrm{J}=2-1$, and $\mathrm{HCO}^{+} \mathrm{J}=1-0$. The region contains a fairly well-defined bipolar outflow, and a $40 \mathrm{~L}_{0}$ infrared source IRAS 0541+0037. The $\mathrm{C}^{18} \mathrm{O}$ emission shows four well-defined condensations, while the ${ }^{13} \mathrm{CO}$ emission is relatively featureless, presumably as a result of its high opacity. The $\mathrm{CS}$ and the $\mathrm{HCO}^{+}$emission each shows a single clump. Impressively enough, as shown in Figure 2, the CS condensation does not coincide with any of the $\mathrm{C}^{18} \mathrm{O}$ peaks or with the $\mathrm{HCO}^{+}$ maximum. Also, it is the CS peak which is coincident with the IRAS source, which is also near the center of the outflow-none of the C180 peaks is close to this position! The ammonia emission studied by Iwata, Fukui and Ogawa (1988) is also highly clumped, with one condensation overlapping, but extending to the east of the CS maximum, and another coincident with the $C^{18} \mathrm{O}$ peak at $\left(-75^{\prime \prime}\right.$, $0 ")$. Two additional $\mathrm{NH}_{3}$ peaks do not correspond to regions of enhanced emission in any of the species we have observed.

Again, I must emphasize that we have observed only a single transition of each species, and cannot eliminate the effect of variations in excitation, and of optical depth, especially for the CS. Nevertheless, it is difficult to believe that these are responsible for all of the difference in the structures that we see. Rather, it seems necessary to confront the fact that $\mathrm{CS}$ and $\mathrm{C}{ }^{18} \mathrm{O}$ - two widely used tracers of cloud structure-give very different pictures of the cloud. The magnitude of the relative abundance variations indicated is on the order of a factor of 10 . If we observed only $\mathrm{C}^{18} \mathrm{O}$, we would simply not be able to make sense of the bipolar flow, while CS alone would give a poor idea of the general cloud structure.

What can be responsible for this differential clumpiness? One possibility is that the chemical evolution in different clumps is in different phases: the time dependence of the abundance of different species (cf. Graedel, Langer, and Frerking 1982) results in an apparent enhancement of different molecules in different condensations at any moment of time. To be verified, this concept would require a great deal more observational data and analysis as well as detailed modeling, but it would be a significant change in our concept cloud structure.
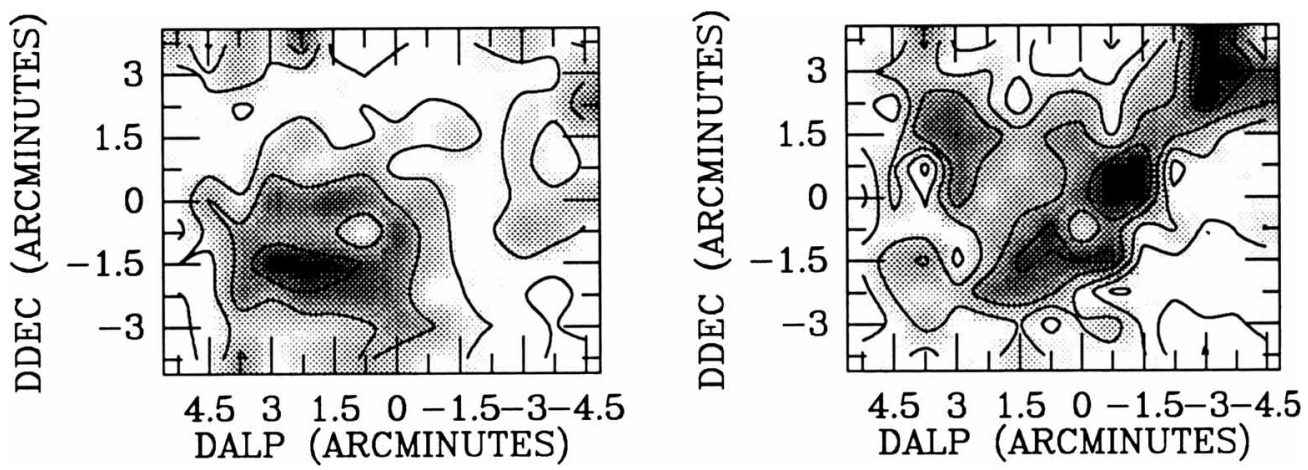

FIG 2. - Maps of CS integrated intensity of $\mathrm{J}=2-1 \mathrm{CS}$ (left) and $\mathrm{J}=1-0 \mathrm{C} 18 \mathrm{O}$ (right) in NGC $2071 \mathrm{~N}$. The $(0,0)$ position is $\alpha=05^{\mathrm{h}} 45^{\mathrm{m}}, \delta=00^{\circ}$ '. The coordinates of IRAS $05451+0037$ are $+120 ",-90 "$ relative to the map center.

Understanding the extent of density inhomogeneities in a cloud and the role that 
they play in their structure is critically dependent on unraveling the issue of molecular abundance variations so that we can properly analyze density structure rather than just nonuniformities in the abundance of trace molecules. Dust continuum emission is subject to variations in physical conditions as well as to systematic uncertainties, but these problems are quite different from those which affect molecular emission. It thus appears highly desirable to use dust emission as a complement to observations of molecules.

Limiting studies to a single, or a few molecular species, even those widely accepted as "good" tracers of molecular material, can lead to serious errors in our perception of the structure of molecular clouds. This is largely the result of significant variations in the abundance of the various tracers within individual clouds. This effect is found in quiescent dark clouds as well as giant molecular clouds, but is particularly prominent in regions where star formation has occurred. In this sense, the apparent complication of molecular abundance variations may prove to be a valuable tracer of the structure and evolutionary state of molecular clouds.

I would like to acknowledge the considerable help of my colleagues Darek Lis, Mike Margulis, Ron Snell, and Bill Irvine in preparing this talk. Astronomy research at FCRAO is supported by the NSF under grant AST 88-15406.

\section{REFERENCES}

Arquilla, R., and Goldsmith, P.F. 1985, Ap.J., 297, 436.

Blake, G.A., Sutton, E.C., Masson, C.R., and Phillips, T.G. 1987, Ap.J., $\underline{315}, 621$.

Dickman, R.L. 1978, Ap.J. Suppl., 37, 407.

Federman, S.R., Huntress, W.T. Jr., and Prasad, S.S. 1990, Ap.J., 354, 504.

Frerking, M.A., Langer, W.D., and Wilson, R.W. 1982, Ap.J., 262, 590.

Goldsmith, P.F., Langer, W.D., Carlson, E.R., and Wilson, R.W. 1980, in Proc.

IAU Symp. 87, B. Andrew, ed. Dordrecht: Reidel, p. 417.

Goldsmith, P.F., Snell, R.L., Hasegawa, T., and Ukita, N. 1987, Ap.J., 314, 525.

Goldsmith, P.F., Margulis, M., Snell, R.L., and Fukui, Y. 1990, in preparation.

Graedel, T.E., Langer, W.D., and Frerking, M.A. 1982, Ap.J.Suppl., 48, 321.

Irvine, W.M., Goldsmith, P.F., and Hjalmarson, A. 1987, in Interstellar Processes, H. Thronson and D. Hollenbach, eds. Dordrecht: Reidel, 561 .

Irvine, W.M. 1990, in Chemistry and Spectroscopy of Interstellar Molecules, N.

Kaifu, ed. Tokyo: University of Tokyo, in press.

Iwata, T., Fukui, Y., and Ogawa, H. 1988, Ap.J., $\underline{325}, 372$.

Johansson, L.E.B. et al. 1984, Astr. Astrophys., 130, 227.

Langer, W.D., Wilson, R.W., Goldsmith, P.F., and Beichman, C.A. 1989, Ap.J., 337, 355.

Lis, D.C., and Goldsmith, P.F. 1989, Ap.J., $\underline{337}, 704$.

Lis, D.C., and Goldsmith, P.F. to appear in Ap.J., 1991.

Minh, Y.C., Irvine, W.M., and Ziurys, L.M. 1988, Ap.J., 334, 175.

Olano, C.A., Walmsley, C.M., and Wilson, T.L. 1988, Astr. Astrophys., 196, 194.

Pineau des Forets, G., Roueff, E., and Flower, D.R. 1989, J. Chem. Soc. Faraday Trans., $\underline{2}, \underline{85(10),} 1665$.

Plambeck, R. and Wright, M. 1988, in Molecular Clouds in the Milky Way and

External Galaxies, R.Dickman, R.Snell, J.Young, eds. Berlin: Springer, 182.

Swade, D.A. 1989, Ap.J. Suppl., 71, 219; Ap.J., 345, 828.

Vogel, S.N., Genzel, R., and Palmer, P. 1987, Ap.J., 316, 243.

Zhou, S., Evans, N.J. II, and Mundy, L. 1990, Ap.J., $\underline{355}, 159$. 